\title{
$\beta$-羰基膦氧化合物的合成研究进展
}

\author{
阮利衡刘畅孙京* 周明东* \\ (辽宁石油化工大学 化学化工与环境学部 抚顺 113001)
}

\begin{abstract}
摘要 $\beta$-羰基膦氧化合物是一类非常重要的有机膦化合物在有机合成化学中具有广泛的用途, 科研工作者一直致力于 开发更加简单、绿色、高效的合成方法. 总结归纳了近年来 $\beta$-羰基膦氧化物合成策略的研究进展，主要概述了通过磷 中心自由基加成反应及水合反应高效制备 $\beta$-羰基膦氧化合物的合成路线.
\end{abstract}

关键词 $\beta$-羰基膦氧化合物; 磷中心自由基; 水合反应

\section{Recent Advances on the Synthesis of $\beta$-Ketophosphine Oxides}

\author{
Ruan, Liheng Liu, Chang Sun, Jing* Zhou, Mingdong* \\ (College of Chemistry, Chemical Engineering and Environment, Liaoning Shihua University, Fushun 113001)
}

\begin{abstract}
Ketophosphine oxides are extremely important phosphorus-containing compounds and widely utilitied in the field of organic synthesis chemistry. Developing simple, green and efficient protocol to synthesize $\beta$-ketophosphine oxides has attracted great attention of chemists in recent years. The recent advances on the synthesis of $\beta$-ketophosphine oxides via phosphorus-centered radical addition reaction or hydration reaction are summarized.

Keywords $\beta$-ketophosphine oxide; phosphorus-centered radical; hydration reaction
\end{abstract}

$\beta$-羰基膦氧化合物作为一类重要的含磷有机化合 物在有机化学、配位化学、生物化学等诸多领域都具有 广泛的应用 ${ }^{[1]}$. 在有机合成中是一类重要的有机合成子, 可以通过一系列转化得到各类重要的衍生物, 是著名的 Horner-Wadsworth-Emmons (HWE) 反应的原料 ${ }^{[2]}$, 也可 以用于制备各类 $\beta$-胺基膦酸、手型 $\beta$-羟基膦酸等化合物 [3]. 在配位化学中 $\beta$-羰基膦氧化合物作为常见的有机膦 配体发挥着重要作用 ${ }^{[4]}$. 此外, $\beta$-羰基膦氧衍生物具有 广泛的生物学活性, 如抗癌功效、促进骨质代谢以及抑 制 $\beta$-内酰胺酶的活性 ${ }^{[5]}$. 鉴于 $\beta$-羰基膦氧化合物的重要 性和应用性，科研工作者一直努力开发高效的合成路线. 目前, $\beta$-羊炭基膦氧化合物的合成方法主要包括 Arbuzov 反

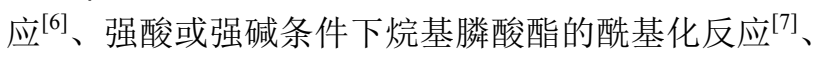

磷中心自由基的加成反应及不饱和膦氧化合物的水合 反应. 近年来, 磷中心自由基的加成反应及水合反应作 为高效、环境友好及原子经济性的合成策略逐渐激发了 广大化学工作者的兴趣. 因此, 本文结合国内外近年来 的研究报道, 基于磷自由基加成反应和水合反应高效制
备 $\beta$-羰基膦氧化合物的反应类型进行了分类、归纳和总 结, 以期待对该领域的发展起到一定的积极作用.

\section{1 磷自由基参与的反应进展}

磷自由基反应在构建 $\pi$-共轭材料、有机膦配体及生 物活性分子等领域占有重要地位 ${ }^{[8]}$. 在磷自由基形成的 过程中, 利用常见有机类自由基引发剂经过氢原子转移 过程(Hydrogen atom transfer, HAT)促使 $\mathrm{P}-\mathrm{H}$ 键均裂是 得到磷中心自由基的方法之一. 另一种得到磷自由基的 方法是氧化活化膦-氢氧化物, 通过单电子转移过程 (Single electron transfer, SET) 及随后在溶剂等作用下的 去质子化形成磷中心自由基(Scheme 1).

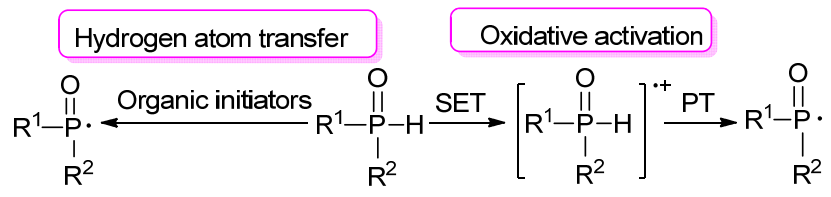

图式 1 磷自由基的引发方式

Scheme 1 Methods for phosphorus-centered radical initiation

* Corresponding authors. E-mail: sunjing@Inpu.edu.cn; mingdong.zhou@Inpu.edu.cn Received January 17, 2019; revised April 15, 2019; published online April 19, 2019. Project supported by the Doctor Startup Fund of Liaoning Province (No. 20180540085). 辽宁省博士科研启动基金(No. 20180540085)资助项目. 


\section{1 氢原子转移得到磷自由基}

有机过氧化合物是常见的氢原子转移试剂, 例如叔 丁基过氧化氢(TBHP)在催化剂的作用下生成叔丁氧自 由基物种，而叔丁氧自由基可以选择性地瞳取膦-氢氧 化物中的氢原子形成磷自由基, 而活性的磷自由基随后 发生各类高效转化. 2015 年, $\mathrm{Zhao}$ 等 ${ }^{[9]}$ 以 $\mathrm{CuSO}_{4} \cdot 5 \mathrm{H}_{2} \mathrm{O}$ 作 催化剂, TBHP 为氧化剂, 氨水作碱, $\alpha$-炔酸化合物 $\mathbf{1}$ 与 膦-氢氧化物在 $60{ }^{\circ} \mathrm{C}$ 乙腈溶液中反应 $2 \mathrm{~h}$, 以优良的产 率得到一系列 $\beta$-羰基膦氧化合物 2 (Scheme 2). 该反应 体系具有反应时间短、催化剂价格低廉和底物普适性好 等优点. 作者经过一系列机理实验提出了反应的可能历 程: 叔丁基过氧化氢在铜催化剂作用下形成叔丁氧自由 基，随后叔丁氧基自由基㩲取膦-氢氧化物的氢原子生 成磷自由基 $\mathbf{A}$, 与此同时苯丙炔酸与二价铜反应形成炔 烃物种 $\mathbf{B}$, 而物种 $\mathbf{A}$ 与 $\mathbf{B}$ 经历自由基加成得到化学中间 体 $\mathbf{C}$, 最后经过脱羧质子化等过程得到目标化合物, 而 且作者认为目标化合物中的羰基氧原子来源于 TBHP.

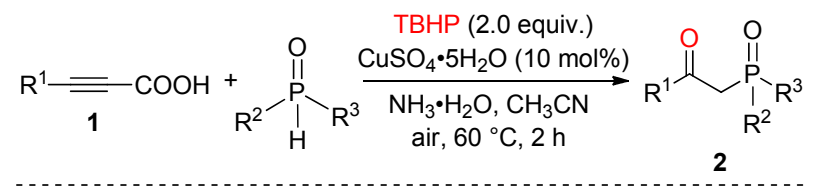

$\mathrm{TBHP} \stackrel{\mathrm{Cu}(\mathrm{II})}{\longrightarrow} t-\mathrm{BuO} \cdot+\cdot \mathrm{OH}$

$\mathrm{Ph}=\mathrm{COOH} \stackrel{\mathrm{Cu}(\mathrm{II})}{\longrightarrow} \mathrm{Ph}=\mathrm{COOCu}(\mathrm{II})$
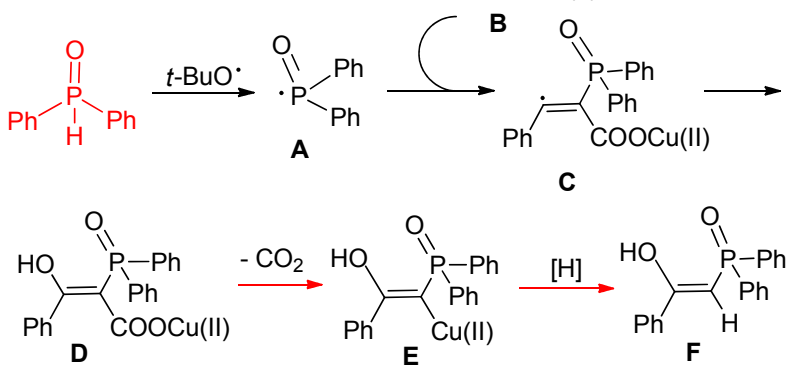<smiles>CC(C)CC(=O)CP(=O)(c1ccccc1)c1ccccc1</smiles>

图式 2 炔酸与膦-氢氧化物串联磷化-脱羧-氧化反应

Scheme 2 Tandem phosphination-decarboxylation-oxidation of alkynyl acids with H-phosphine oxides

基于 $\mathrm{CuSO}_{4} \cdot 5 \mathrm{H}_{2} \mathrm{O} / \mathrm{TBHP}$ 的催化体系, She 等 ${ }^{[10]}$ 报 道了在温和的反应条件下硫酸铜催化的烯丙醇化合物 3、膦-氢氧化物及 TBHP 的三组分氧化磷酰化，高效地 制备了一系列带有不同官能团的 $\beta$-羰基膦氧化合物 4 (Eq. 1). 其中, $\beta$-羰基膦氧化合物中的羰基氧原子来源 于 TBHP, 在该反应中 TBHP 不仅作为膦-氢氧化物的自 由基引发剂, 而且是反应体系的偶联组分.

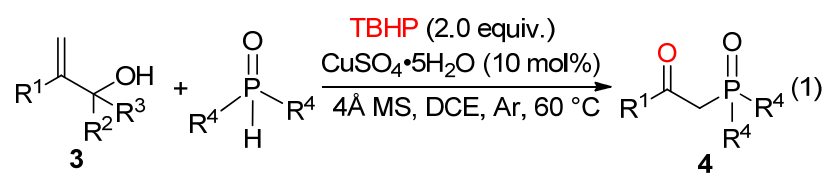

2015 年, Radivoy 课题组 ${ }^{[11]}$ 报道了在空气条件且无 其他额外添加剂和配体的作用下，负载在 $\mathrm{ZnO}$ 上的纳 米铜 $(\mathrm{CuNPs})$ 催化炔烃 $\mathbf{5}$ 与膦-氢氧化物合成 $\beta$-羊炭基膦酸 酯类化合物的反应(Scheme 3). 在该反应体系中，若原 料是 $\alpha$-芳基炔类化合物则会经过自由基加成得到 $\beta$-羰 基膦氧化合物 $\mathbf{6}$; 若原料是脂肪炔则经过反马式加成形 成 $\alpha$-烯基膦酸酯化合物 7; 而对于烯烃类化合物无论是 芳香类烯烃或脂肪类烯烃均可得到 $\beta$-羰基膦氧化合物.

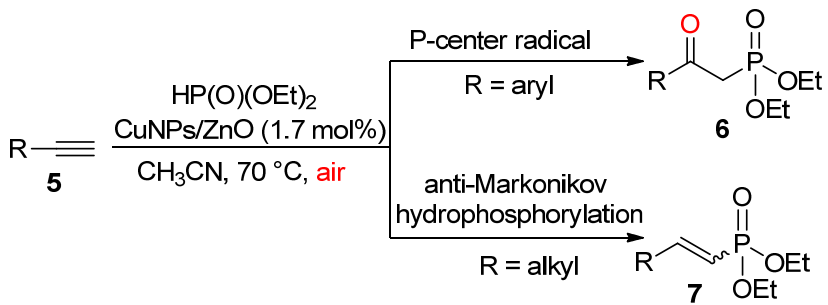

图式 3 负载在 $\mathrm{ZnO}$ 上的纳米铜 $(\mathrm{CuNPs})$ 催化炔烃与膦-氢氧 化物合成 $\beta$-羰基膦酸酯类化合物

Scheme 3 Synthesis of $\beta$-ketophosphonates from alkynes with $\mathrm{H}$-phosphine oxides catalyzed by $\mathrm{CuNPs} / \mathrm{ZnO}$

2016 年, Wei 等 ${ }^{[12]}$ 报道了在 $\mathrm{O}_{2}$ 氛围下, $\mathrm{Cu}(\mathrm{I})$ 催化炔 烃 8 和膦-氢氧化物的直接氧化磷酰化反应, 得到 $\beta$-羰 基膦氧化物 9 (Scheme 4), 该反应在温和的条件下，不 需要碱和其他的催化剂即可发生. 反应机理表明：在 $\mathrm{Cu}(\mathrm{I})$ 及氧气的共同作用下，膦一氢氧化物形成磷自由基， 然后磷自由基与炔烃发生选择性加成，并经过互变异构 得到目标产物.
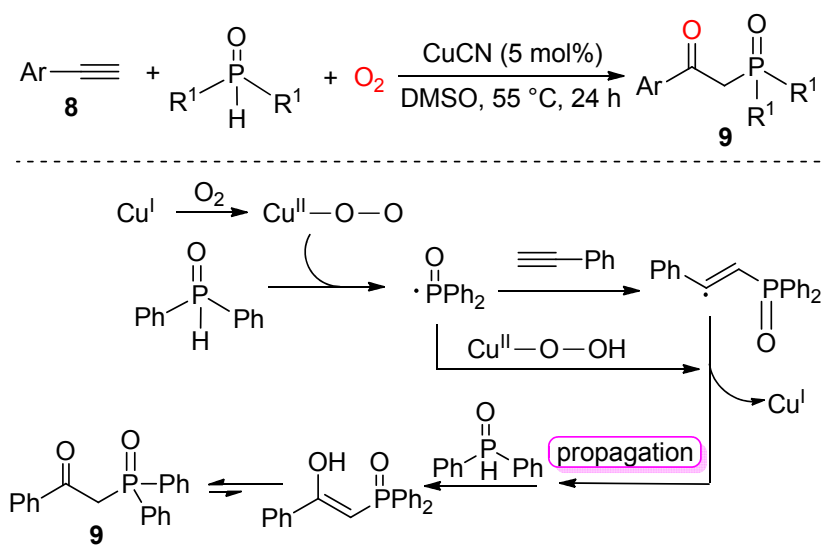

图式 4 铜催化炔烃和膦-氢氧化物的直接氧化磷酰化反应 Scheme 4 Copper-catalyzed oxyphosphorylation of alkynes and H-phosphine 
随后, Zhao 课题组 ${ }^{[13]}$ 报道了以 $\mathrm{CuSO}_{4} \cdot 5 \mathrm{H}_{2} \mathrm{O}$ 为催化 剂, 烯基酸化合物或烯烃为原料, “一锅法”合成了 $\beta$-羰 基膦氧化合物 11. 在该反应体系中铜盐、乙腈及氧气形 成了一种活性氧化物 $\left[(\mathrm{MeCN})_{n} \mathrm{Cu}\right.$ II-O-O·], 其催化膦-氢 氧化物形成了磷自由基物种. 作者通过对目标化合物中 的羰基氧原子的来源进行研究, 认为氧原子来源于氧气 (Eq. 2).

$$
\begin{aligned}
& \underbrace{\mathrm{R}^{1}}_{\mathbf{1 0}} \\
& \mathrm{R}=\text { aryl, heterocyclic; } \mathrm{X}=\mathrm{COOH}, \mathrm{H} \\
& \mathrm{R}^{1}=\mathrm{H}, \mathrm{CH}_{3} ; \mathrm{R}^{2}=\text { aryl, aryloxy, alkoxy }
\end{aligned}
$$

\section{2 “单电子转移” 得到磷自由基}

在各种过渡金属催化剂如银盐、铜盐、锰盐等及可 见光作用下, 膦-氢氧化物能够经历单电子转移失去一 个电子得到膦-氢氧化物正离子中间体, 随后在溶剂等 作用下失去质子从而形成了磷自由基, 而磷自由基能够 发生后续的一系列转化过程.

\subsection{1 银盐为磷自由基引发剂}

银盐作为一种廉价高效的催化剂被用于磷自由基 加成反应制备 $\beta$-羊炭基膦酸酯化合物. 2015 年, Wang 课题 组 ${ }^{[14]}$ 报道了碳酸银催化苯炔酸类化合物 12 与膦-氢氧 化物以空气为氧化剂的脱羧偶联反应, 合成了 $\beta$-羰基膦 氧化合物 13 (Eq. 3). 作者采用了同位素标记实验验证 了目标产物中的氧原子来源于空气中的氧气. 随后, Zhao 课题组 ${ }^{[15]}$ 首次在 $\mathrm{Ag} / \mathrm{Cu} / \mathrm{K}_{2} \mathrm{~S}_{2} \mathrm{O}_{8}$ 的催化体系下, 以 炔烃为原料 “一锅法” 高效地合成了一系列 $\beta$-羰基膦酸 酯化合物(Eq. 4). 该反应体系操作简单、条件温和、底 物普适性好、且起始原料简单, 令反应具有良好的应用 前景. 此外, 其他研究小组基于银催化的膦-氢化合物 的自由基加成反应体系合成了 膦酰化香豆素衍生 物 ${ }^{[16] 、} \beta$-羰基膦酰化氮杂螺环化合物 ${ }^{[17,18]} 、 \beta$-羰基膦酰 化吲哚类化合物 ${ }^{[19]}$ 及膦酰化 2-羰基-3,4-二氢喹啉衍生 物 ${ }^{[20]}$.
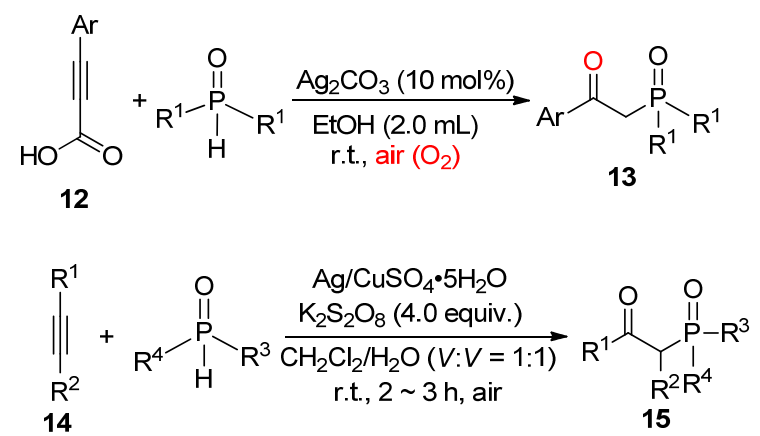

$\mathrm{R}^{1}=$ aryl, alkyl; $\mathrm{R}^{2}=\mathrm{H}, \mathrm{Me} ; \mathrm{R}^{3}, \mathrm{R}^{4}=$ aryl, aryloxy, alkoxy
2017 年, Dong 和 Peng 等 ${ }^{[21]}$ 成功地实现了银催化的 1,3-二羰基化合物 $\mathbf{1 6}$ 与 $\mathrm{H}-$ 膦酸酯类化合物的 $\mathrm{C}\left(\mathrm{sp}^{3}\right)-\mathrm{H} /$ $\mathrm{P}-\mathrm{H}$ 交叉偶联反应，合成了 $\beta$-羰基膦氧化合物 17 (Scheme 5). 该反应体系避免了碱和配体的参与, 具有广 泛的底物普适性、专一的选择性及良好的官能团兼容性 等优点. $\mathrm{Qu}$ 等 ${ }^{[22]}$ 报道了微波条件下银催化的 $\beta$-芳基- $\alpha, \beta$ 不饱和羰基化合物的自由基区域性磷酸化反应，成功地 得到了各类 $\beta$-羰基-trans-膦酰化烯烃化合物.

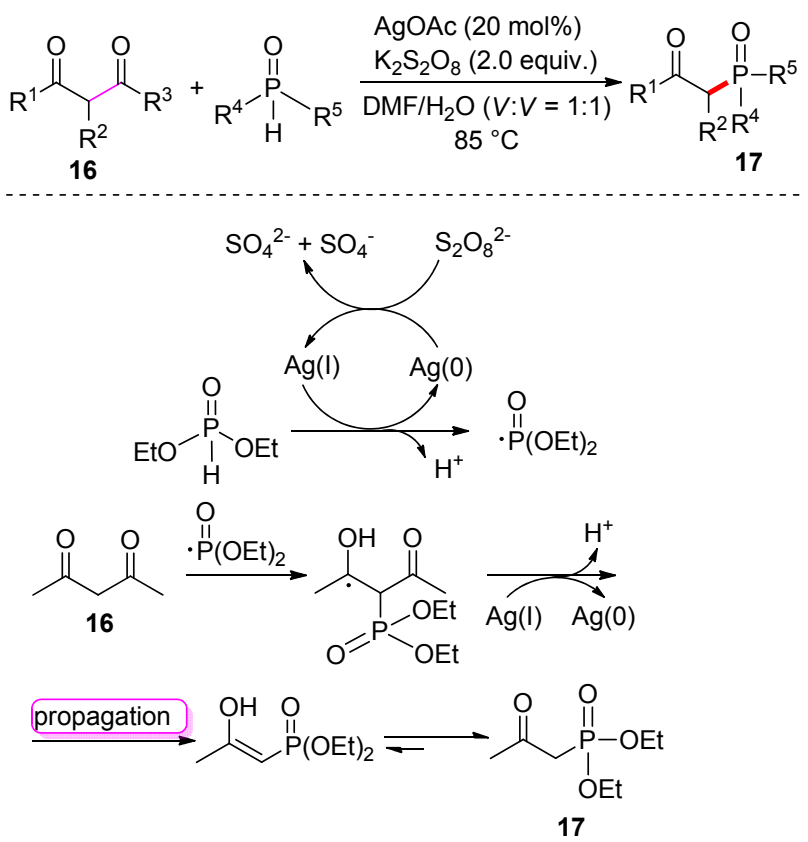

图式 5 银催化 1,3-二羰基化合物与 $\mathrm{H}$-膦酸酯类化合物的 $\mathrm{C}\left(\mathrm{sp}^{3}\right)-\mathrm{H} / \mathrm{P}-\mathrm{H}$ 的交叉偶联反应

Scheme 5 Silver-catalyzed $\mathrm{C}\left(\mathrm{sp}^{3}\right)-\mathrm{H} / \mathrm{P}-\mathrm{H}$ cross-coupling of 1,3-dicabonyl compounds with H-phosphonates

\subsection{2 铜/铁盐为磷自由基引发剂}

2011 年, $\mathrm{Ji}$ 等 ${ }^{[23]}$ 报道了在 $\mathrm{O}_{2}$ 氛围下, $\mathrm{CuBr}_{2} / \mathrm{FeBr}_{3}$ 催 化烯烃 18 与含 $\mathrm{H}$-亚膦酸酯的氧化磷酰化反应, 合成了 $\beta$-羊基膦酸酯类化合物 19 (Eq. 5). 该反应不需要在低温 下进行且不需要添加化学计量的有机金属试剂. $\mathrm{He}$ 等 ${ }^{[25]}$ 报道了利用 $\mathrm{Cu}(\mathrm{II}) / \mathrm{Fe}(\mathrm{III})$ 的催化体系催化端炔化合物 20 的氧化磷酰化反应，经过自由基历程合成了 $\beta$-羰基膦氧 化物 21 (Scheme 6). 机理研究实验表明, 在 $\mathrm{Cu}(\mathrm{II}) / \mathrm{Fe}(\mathrm{III})$ 的反应体系中, 目标产物中的羰基氧原子来源于 $\mathrm{O}_{2}$. 而 且此反应体系具有官能团兼容性高与实验操作简单等特 点. 与此同时, Chen 等 ${ }^{[24]}$ 研究了 $\mathrm{CuBr}_{2} / \mathrm{FeBr}_{3}$ 体系下通过 无溶剂方法制备各类 $\beta$-羰基膦酸酯化合物.

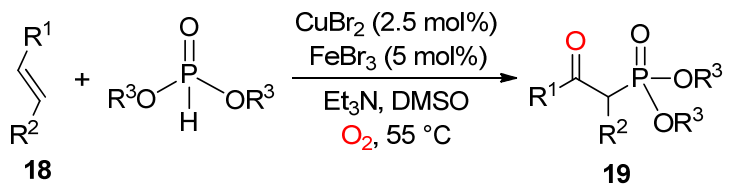




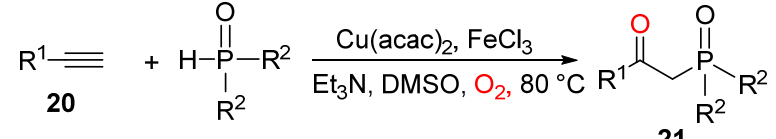
$\mathrm{R}^{1}=$ aryl, heterocyclic; $\mathrm{R}^{2}=$ aryl, alkoxy

21

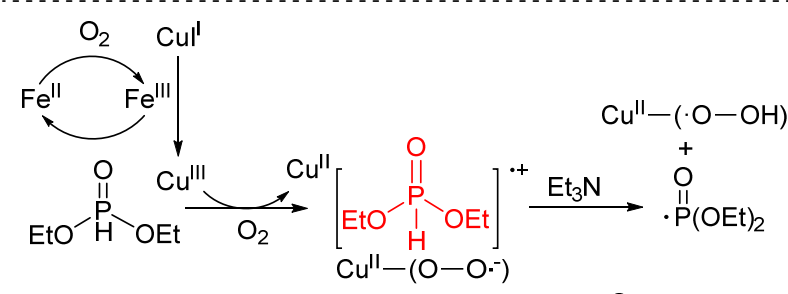

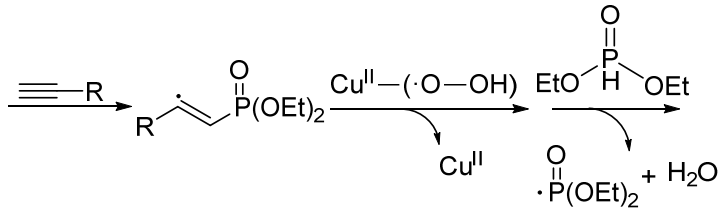<smiles>[R]C(O)=C[P+](=O)OCCC</smiles>

图式 $6 \mathrm{Cu}(\mathrm{II}) / \mathrm{Fe}(\mathrm{III})$ 催化端炔化合物的氧化磷酰化反应 Scheme $6 \mathrm{Cu}(\mathrm{II}) / \mathrm{Fe}(\mathrm{III})$-catalyzed oxyphosphorylation of terminal alkynes

2015 年, Song 课题组 ${ }^{[26]}$ 报道了在 $\mathrm{Cu}(\mathrm{I}) / \mathrm{Fe}(\mathrm{III})$ 盐为
共同催化剂的作用下，以芳基醛化合物 22 为初始原料 通过 “一锅法” 合成了一系列 $\beta$-羰基膦氧化合物 24. 随 后, Song 等 ${ }^{[27,28]}$ 相继报道了以苯炔或苯炔酸化合物 $\mathbf{2 5}$, $\alpha, \beta$-不饱和酯类化合物和 $\alpha, \beta$-不饱和酰胺类化合 26 为原 料制备了一系列 $\beta$-羰基膦氧化合物. 经过机理研究, 作 者认为该反应体系涉及自由基反应历程，且产物中的羰 基氧原子均来源于氧气. 2016 年, Song 等 ${ }^{[29]}$ 报道了以 $\alpha, \beta$-不饱和羰基化合物 27 为原料制备各类 $\beta$-羊炭基膦氧 化物. 该反应体系通过取代基控制反应的化学选择性, 再经自由基反应历程后得到目标化合物，同位素标记实 验表明经历 path $\mathrm{A}, \beta$-羰基膦氧化物分子中的羰基氧原 子来源于反应原料且反应体系伴随有副产物醛的生成 (Scheme 7).

2017 年, $\mathrm{Cai}$ 等 ${ }^{[30]}$ 报道了不同反应温度控制的 $\mathrm{Cu}(\mathrm{I}) /$ $\mathrm{Fe}(\mathrm{III})$ 催化苯乙烯类化合物 $\mathbf{3 0}$ 与含 $\mathrm{H}$-亚膦酸酯的磷酸 化反应和氧化磷酸化反应(Scheme 8). 该反应体系为自 由基反应历程，在反应温度为 $110{ }^{\circ} \mathrm{C}$ 时，自由基中间体 直接氧化去质子化得到烯基膦氧化合物 31; 而在反应 温度为 $90{ }^{\circ} \mathrm{C}$ 时，自由基中间体继续与羟基自由基反应 再发生氧化得到 $\beta$-羊炭基膦氧化合物 32 .

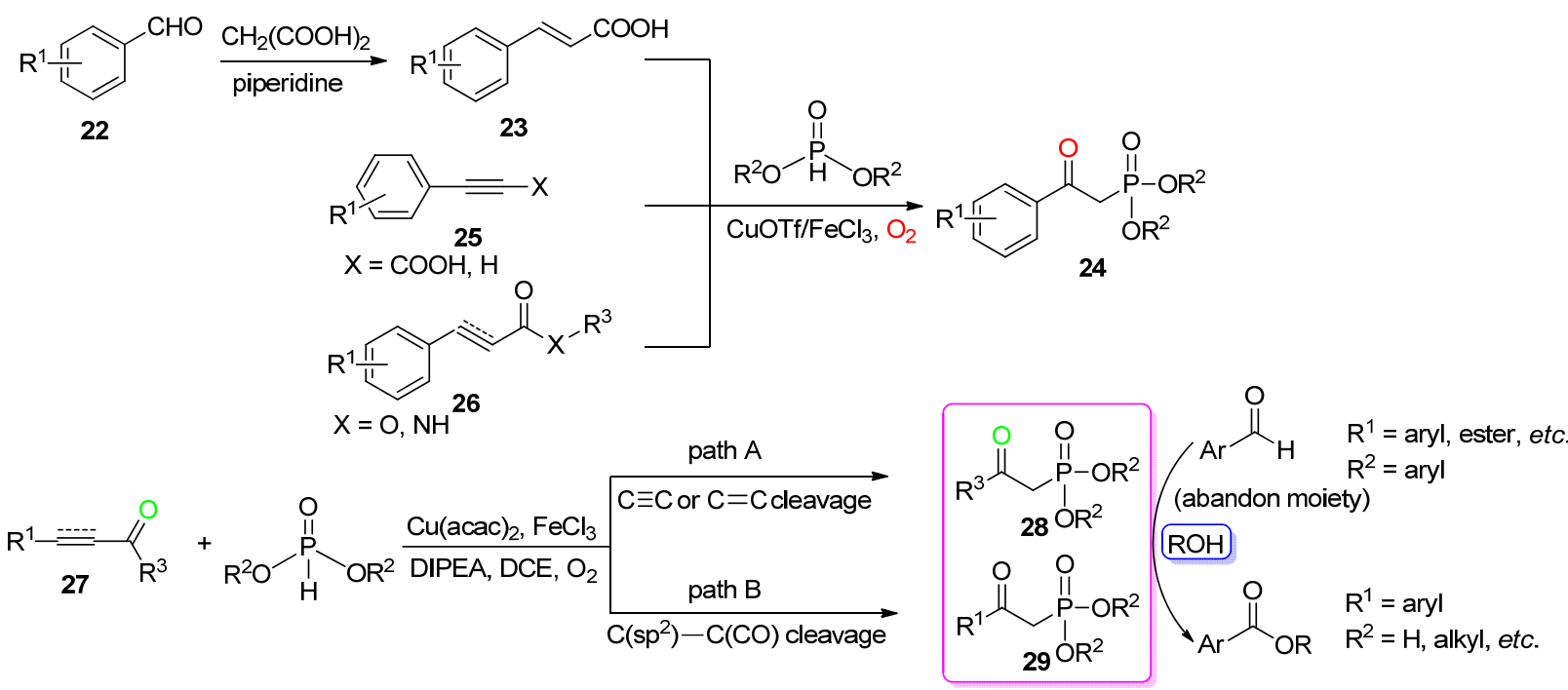

图式 $7 \mathrm{Cu} / \mathrm{Fe}$ 共催化 $\beta$-羰基膦氧化物的合成

Scheme 7 Synthesis of $\beta$-ketophosphonate oxides catalyzed by $\mathrm{Cu} / \mathrm{Fe}$ catalysis

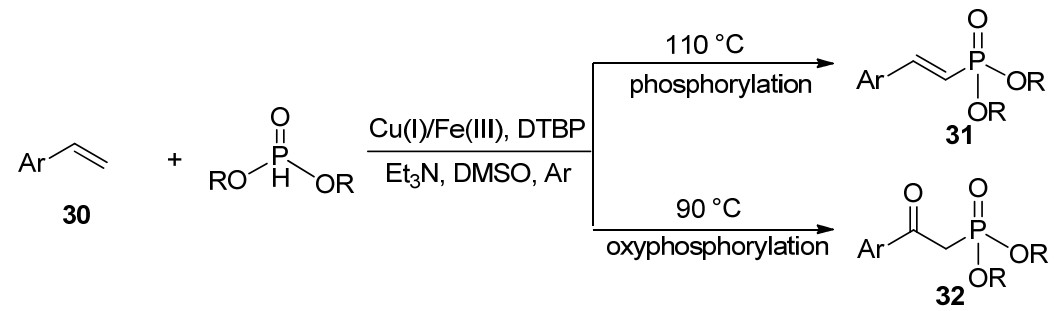

图式 $8 \mathrm{Cu}(\mathrm{I}) / \mathrm{Fe}(\mathrm{III})$ 催化苯乙烯的氧化磷酸化反应合成 $\beta$-羰基膦氧化合物 Scheme $8 \mathrm{Cu}(\mathrm{I}) / \mathrm{Fe}(\mathrm{III})$-catalyzed oxyphosphorylation of alkenyls for the synthesis of $\beta$-ketophosphonates 
单一的铜盐催化体系制备 $\beta$-羰基膦氧化合物的反 应也有相关研究. Lei 等 ${ }^{[31]}$ 报道了在 $\mathrm{N}_{2}$ 氛围下, $\mathrm{CuCl}$ 作 催化剂、 $\mathrm{PCy}_{3}$ 为配体在二氧六环溶剂中芳基酮 $O$-乙酰 肜类化合物 33 与含 $\mathrm{H}$ 膦氧化物的 $\alpha$-磷酰化反应, 成功 地合成了一系列 $\beta$-羰基膦氧化物 34 (Eq. 6). 该反应体 系实现了铜催化的自由基 $\mathrm{C}\left(\mathrm{sp}^{3}\right)-\mathrm{H}$ 与自由基 $\mathrm{P}-\mathrm{H}$ 的 自由基交叉偶联反应，作者通过电子顺磁共振(EPR)实 验探索了初步的反应机理, 并提出酮- $O$-乙酰肜类化合 物经历还原过程产生的亚胺离子经过异构化形成 $\alpha$-sp ${ }^{3}$ 碳自由基, 其与膦氢氧化物经氧化生成的磷自由基发生 自由基偶联反应，随后经过取代反应/水解反应得到目 标化合物.

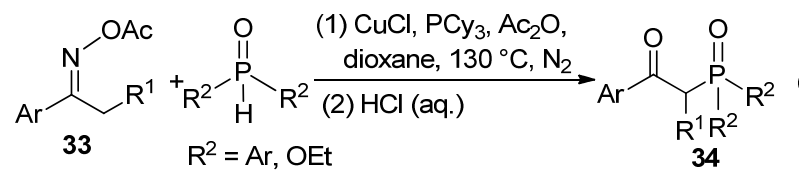

2017 年, $\mathrm{Wei}$ 等 ${ }^{[32]}$ 报道了在 $\mathrm{O}_{2}$ 条件下, $\mathrm{Cu}$ 催化的烯 酰胺类化合物 35 与含 $\mathrm{H}-$ 膦氧化物的直接氧化磷酰化反 应, 合成了 $\beta$-羰基膦氧化合物(Eq. 7). 该反应体系不仅 条件温和、操作简单方便, 同时也具有良好的底物普适 性. 随后, Wei 等 ${ }^{[33,34]}$ 报道了在不同铜催化剂的催化作 用下，以酮类化合物 37 作原料合成 $\beta$-羊炭基膦氧化合物 的反应. 而且该类反应体系得到的目标产物中的羰基氧 原子均来源于氧气(Scheme 9).

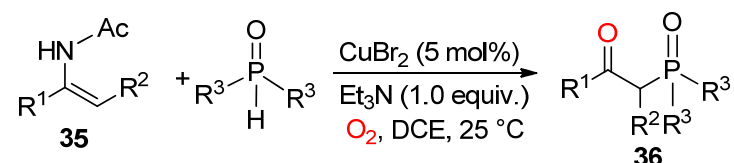

$\mathrm{R}^{1}=$ Aryl, heterocycle; $\mathrm{R}^{2}=\mathrm{H}, \mathrm{CH}_{3} ; \mathrm{R}^{3}=\mathrm{Ph}, \mathrm{OEt}, \mathrm{OMe}, \mathrm{OBu}$

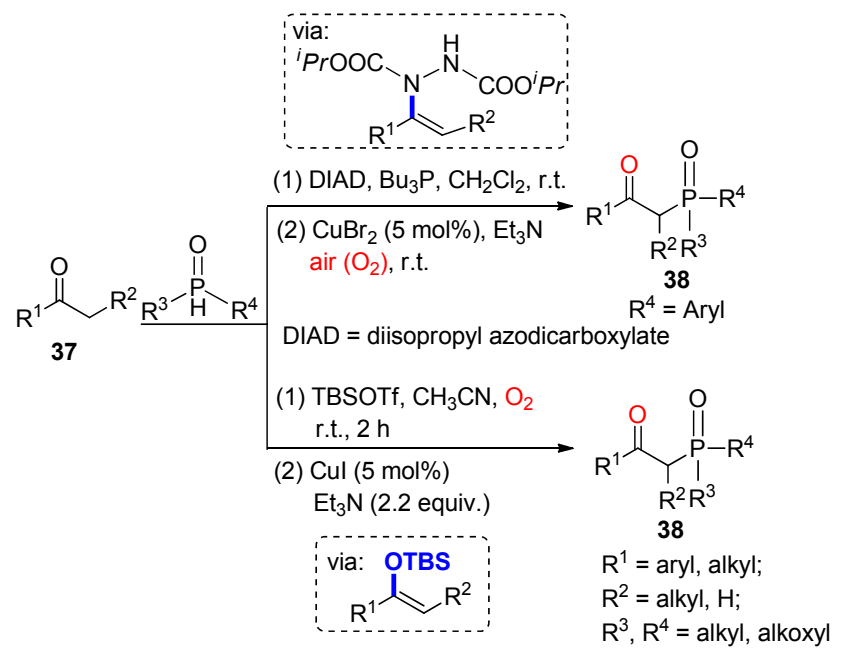

图式 9 铜催化酮与膦-氢化合物的氧化偶联反应

Scheme 9 Cu-catalyzed oxidative coupling of ketones with $\mathrm{P}(\mathrm{O})-\mathrm{H}$

2017 年, Wang 等 ${ }^{[35]}$ 报道了铜催化苯乙烯 30 与含
$\mathrm{H}$-亚膦酸酯在不同氧化条件下的反应(Scheme 10). 当 以 $\mathrm{K}_{2} \mathrm{~S}_{2} \mathrm{O}_{8}$ 为氧化剂时，能够得到烯基膦氧化合物 39; 而 选取氧气作氧化剂时, 则高效地得到了 $\beta$-羊炭基膦氧化合 物 40. 研究表明, 在该铜催化的氧化膦酰化偶联体系中, 溶剂具有重要作用，乙腈不仅是良好的溶剂而且起到配 体作用，与此同时反应体系中乙腈可以回收循环使用.

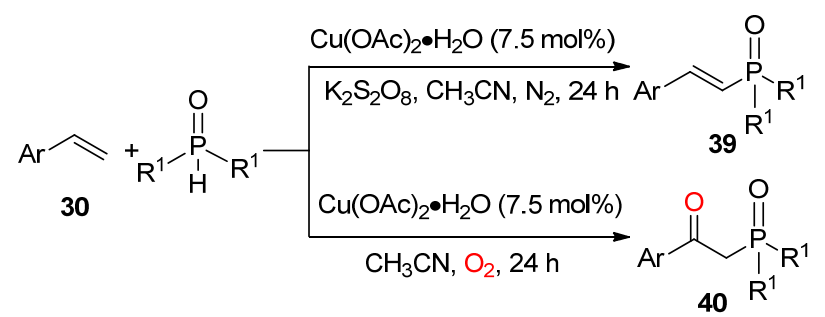

图式 10 铜催化苯乙烯与膦氢化合物的氧化偶联反应 Scheme $10 \mathrm{Cu}$-catalyzed oxidative coupling of styrenes with $\mathrm{P}(\mathrm{O})-\mathrm{H}$

\subsection{3 锰盐为磷自由基引发剂}

三价锰盐作为单电子氧化剂, 在自由基化学领域得 到广泛应用 ${ }^{[33]}$. 在三价锰盐的作用下膦-氢氧化物能够 生成磷自由基, 然后发生后续的高效转化. 而在其他一 些反应体系中，三价锰盐还扮演着氧化剂的角色，往往 需要使用化学计量的锰盐. 2010 年, Zou 等 ${ }^{[37]}$ 报道了 $\mathrm{Mn}(\mathrm{III})$ 促进的 $\alpha, \beta$-不饱和芳基酮类化合物 $\mathbf{4 1}$ 的磷酸化 反应，高效地合成了烯烃膦酸酯类化合物 42 (Scheme 11). 芳基烯烃的 $\alpha$ 位连有酮、酰胺、硝基、酯基等共轭 结构的基团时，也可以高效地完成反应. 同时该反应体 系也适合由芳炔烃类化合物 $\mathbf{4 3}$ 制备 1-狮酮类化合物及 其衍生物 44. 随后, Zou 等 ${ }^{[38,39]}$ 报道了 $\mathrm{Mn}(\mathrm{III})$ 引发的磷 自由基制备 3-芳基-2-膦酰基-1-䒢酮类化合物、3-膦酰基 黄酮类化合物及 3-膦酰基香豆素类化合物.

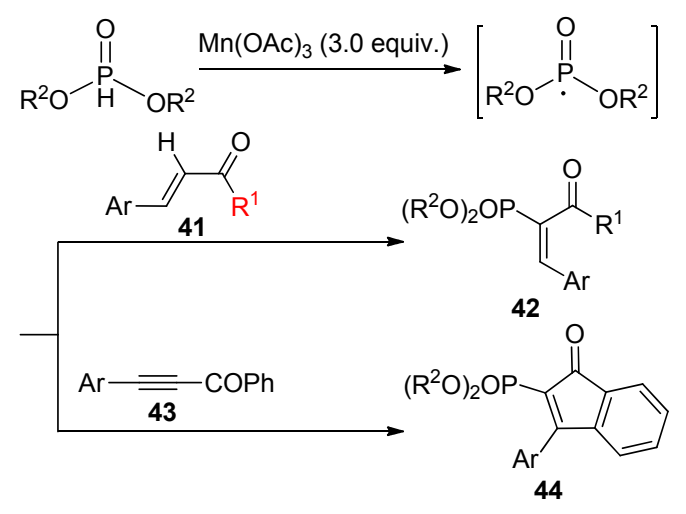

图式 $11 \mathrm{Mn}(\mathrm{III})$ 促进芳烯烃、芳炔烃的直接磷酸化反应 Scheme $11 \mathrm{Mn}(\mathrm{III})$-mediated direct phosphonation of arylakenes and arylalkynes

2016 年, Zou 等 ${ }^{[40]}$ 报道了 $\mathrm{Mn}(\mathrm{III})$ 催化的苯乙烯类化 合物的自由基氧化膦酰化反应制备了一系列 $\beta$-羰基膦 
氧化物(Eq. 8). 2017 年, $\mathrm{Yu}$ 等 ${ }^{[41]}$ 报道了以 $N, N$-二甲基烯 胺酮类化合物与含 $\mathrm{H}$ 膦氧化物为原料, 在 $\mathrm{Mn}(\mathrm{OAc})_{3}$ 的 作用下, 经过 $\mathrm{C}\left(\mathrm{sp}^{2}\right)-\mathrm{C}\left(\mathrm{sp}^{2}\right)$ 和 $\mathrm{P}-\mathrm{H}$ 的交叉偶联反应制 备了 $\beta$-羰基膦氧化物(Eq. 9). 该反应产物产率高, 反应 操作简单，底物普适性好且适合大量制备. 随后，Yu 等 ${ }^{[42]}$ 报道了 $\mathrm{Mn}(\mathrm{III})$ 催化乙烯基叠氮化物合成高产率、官 能团兼容性高的 $\beta$-羰基膦氧化物(Eq. 10). 与其他反应 不同的是, 该反应体系避免了 $\mathrm{O}_{2}$ 、强碱、共催化体系及 化学计量的催化剂的使用.

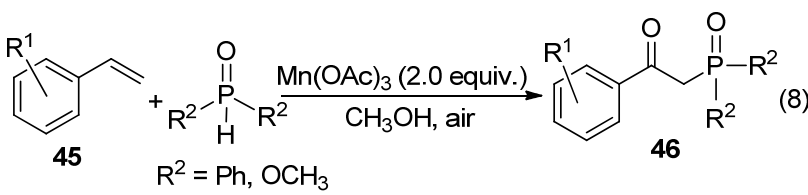

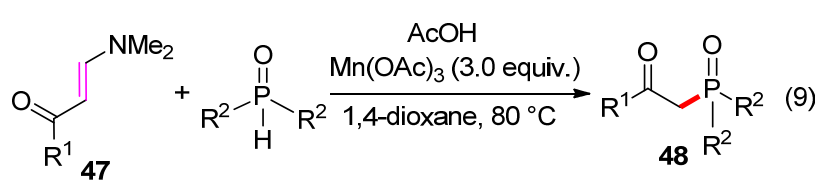<smiles>[R10][R17](=C)C(=C)N</smiles>

\section{2 .4 光催化剂为磷自由基引发剂}

近年来, 光诱导催化的自由基反应已成为有机合成 化学的研究热点之一. 在光催化剂为磷自由基引发剂合 成 $\beta$-羰基膦氧化物的反应中，这些反应体系成功地避免 了金属试剂的使用, 在温和条件下即可顺利进行, 更加 符合绿色化学的理念. $\mathrm{Cai}$ 等 ${ }^{[43]}$ 报道了在温和条件下以 可见光(white LED)及有机染料(Rhodamine B)为催化剂 催化芳炔烃和二芳基膦氧化物的氧化偶联反应，合成了 各种 $\beta$-羰基膦氧化物(Scheme 12). 该反应避免了金属试
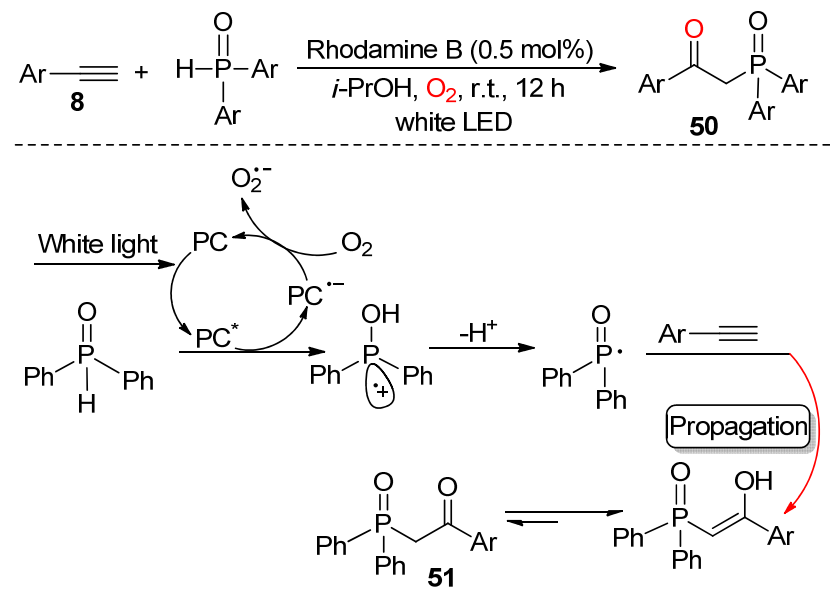

图式 12 可见光催化芳炔烃的膦酸化反应

Scheme 12 Visible-light-induced phosphinylation of aryl alkynes
剂的使用并抑制了其他副产物的生成.

2017 年, $X u$ 等 ${ }^{[44]}$ 报道了在温和条件下, 可见光促进 炔酮类化合物的双官能团化反应，合成了 3-膦酰基香豆 素类化合物(Eq. 11). 在该反应体系中, Eosin $\mathrm{Y}$ 为光催 化剂, TBHP 为氧化剂, 通过自由基串联磷酸化反应和 环化反应合成目标产物. 2018 年, Zou 等 ${ }^{[45]}$ 报道了可见 光诱导的无金属参与的 $\alpha$-烯基羧酸与含 $\mathrm{H}$ 的二芳基膦 氧化物在光催化剂(Rose Bengal)的催化作用下合成 $\beta$-羰 基膦氧化物(Eq. 12). 随后, Zhu 等 ${ }^{[46]}$ 报道了在可见光及 氧气氛围下、无金属参与的烯烃的氧化膦酰化反应，高 效地合成了 $\beta$-羰基膦氧化物(Eq. 13). 与之前的合成方 法相比，光反应的合成方法成功避免了金属试剂参与， 使反应条件温和，更加绿色经济.

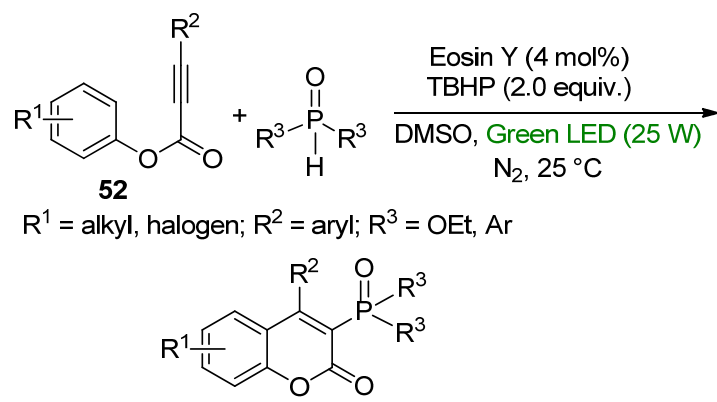

53
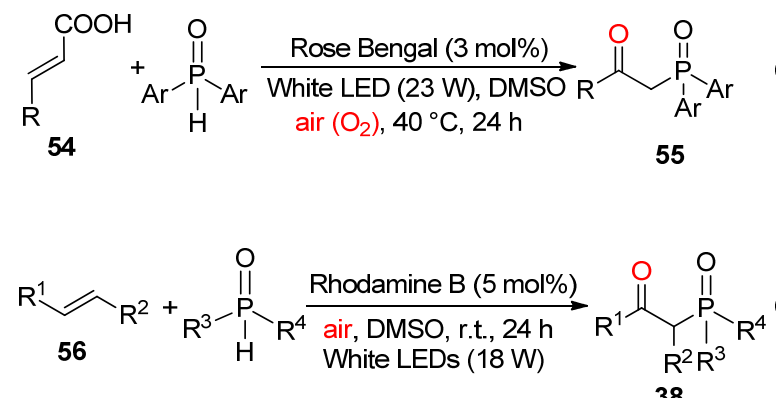

2016 年, Lei 等 ${ }^{[47]}$ 报道了在无过渡金属及其他的有 机自由基引发剂的存在下, $\mathrm{O}_{2}$ 诱导烯烃、炔烃类化合物 与含 $\mathrm{H}$ 膦氧化物发生 $\mathrm{P}-\mathrm{H}$ 键活化反应, 生成了 $\beta$-含氧 膦氧化物(Scheme 13). 机理实验表明 $\mathrm{HP}(\mathrm{O}) \mathrm{Ph}_{2}$ 作为还 原剂促进了氧化磷酰化反应. 值得注意的是：(1)与其他 报道 ${ }^{[0 ~ 13]}$ 形成磷中心自由基的方式不同，在该反应中磷 自由基是由单电子氧化 $\mathrm{P}-\mathrm{H}$ 化合物失去质子得到; (2) 在该反应中苯嗍酸可以促进炔烃化合物的自由基氧化 磷酰化反应, 得到 $\beta$-羰基膦氧化物. 该反应体系避免了 银、铁、铜、锰等过渡金属的使用及副产物的生成.

\section{2 水合作用}

在有机合成中炔烃化合物的水合反应是合成羰基 


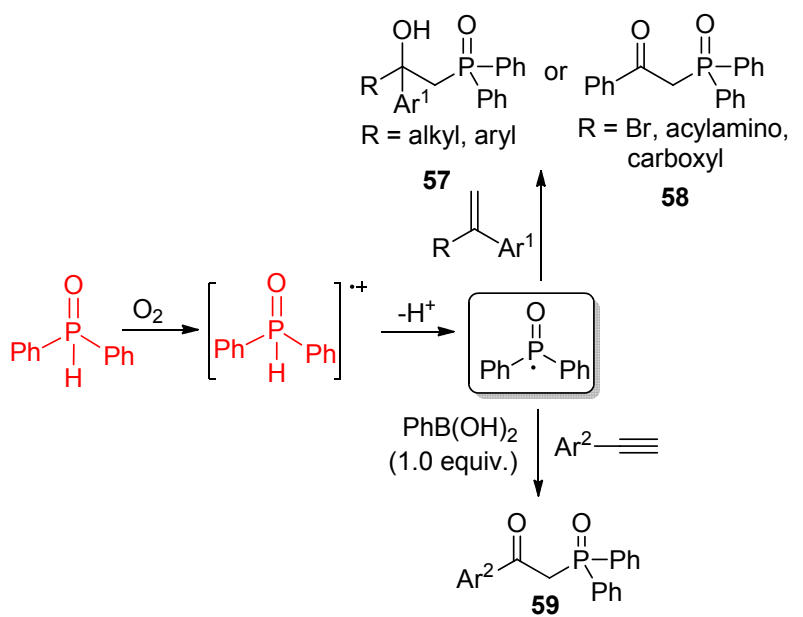

图式 13 烯烃、炔烃的自由基氧化磷酰化反应 Scheme 13 Radical oxyphosphorylation of alkenes and alkynes 化合物的重要方法 ${ }^{[48]}$, 基于不饱和烃通过水合作用直 接引入羰基的策略，一些科研工作者利用不饱和的膦氧 化合物高效地制备了一系列 $\beta$-羊炭基膦氧化合物.

\section{1 过渡金属催化}

1966 年, Sturtz 等 ${ }^{[49]}$ 首次报道录盐高效催化炔基膦 酸酯化合物得到 $\beta$-羊炭基膦酸酯, 由于该反应需要使用有 毒的录盐, 因此科研工作者致力于开发更加简单高效低 毒的催化体系. 2012 年, Zhao 等 ${ }^{[50]}$ 报道了在 Pd(II)催化 炔基膦氧化合物的直接水合作用制备 $\beta$-羰基膦氧化合 物(Eq. 14), 该反应体系避免了酸、碱等助催化剂以及高 毒的录催化剂的使用, 使反应体系趋于绿色环保. $\mathrm{He}$ 等 ${ }^{[51,52]}$ 相继开发了 $\mathrm{Au} 、 \mathrm{Ag}$ 等过渡金属的催化体系, 以 炔基膦酸酯为原料通过水合作用合成 $\beta$-羰基膦酸酯类 化合物，且该类反应体系更加温和，具有更好的 “原子经 济性” 和广泛的底物普适性(Eqs. 15, 16). 同时, Liu 等 ${ }^{[53]}$ 研究了以相类似 $\mathrm{XPhosAuSbF}_{6}$ 为催化剂的水合反应.
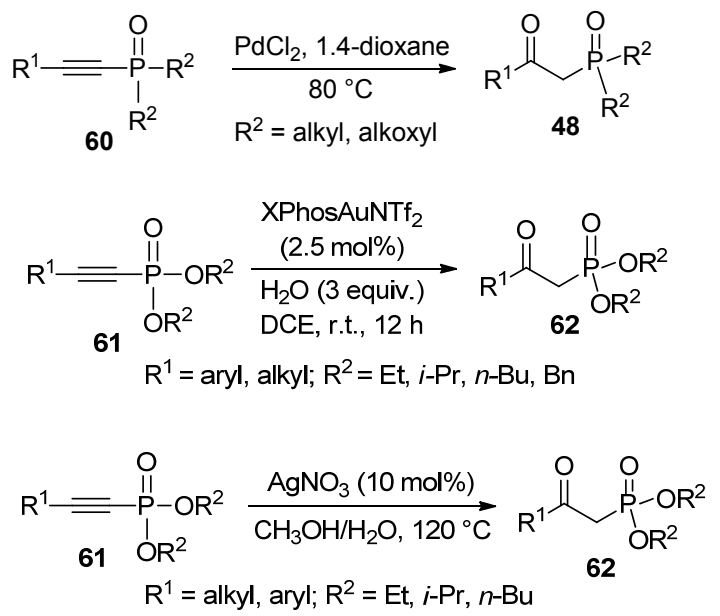

我们课题组 ${ }^{[54,56]}$ 以膦氧联烯化合物为原料开展了
高效合成 $\beta$-羰基膦氧化合物相关方面的工作. 2015 年, 我们课题组 ${ }^{[54]}$ 首次报道了甲基三氧化铼(MTO)为催化 剂, $\mathrm{H}_{2} \mathrm{O}_{2}$ 为氧化剂的条件下, 通过控制 1,2-二苯基膦氧 联烯化合物的区域选择性，得到 $\beta$-羰基- $\gamma$-差基的二苯基 膦氧化合物(Eq. 17).

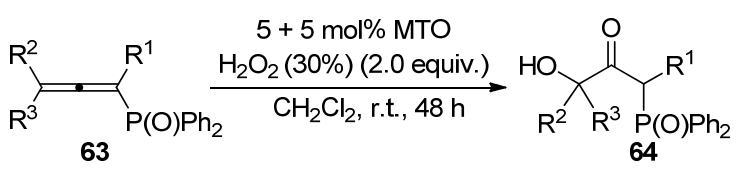

\section{2 非金属催化}

2016 年, Zhu 等 ${ }^{[55]}$ 报道了在离子液体且无金属催化 剂的条件下合成 $\beta$-羰基膦酸酯类化合物(Eq. 18). 在该 反应中避免了有毒及价格昂贵的金属催化剂的使用，离 子液体可以循环使用且官能团兼容性好，产率优异，使 得该反应体系更加符合 “绿色化学” 的理念，为 $\beta$-羰基 膦酸酯的制备提供了一个新的绿色、高效的合成方案.

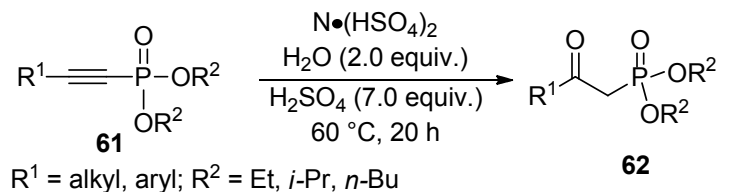

$\mathrm{R}^{1}=$ alkyl, aryl; $\mathrm{R}^{2}=\mathrm{Et}, i-\mathrm{Pr}, n-\mathrm{Bu}$

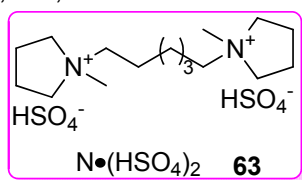

2018 年, 我们课题组 ${ }^{[56]}$ 报道了在温和的反应条件 下以简单的有机碱吡咯烷为催化剂，1,2-二苯基膦氧联 烯化合物通过直接水合作用生成了一系列 $\beta$-羰基膦氧 化合物(Scheme 14). 该反应体系具有反应条件温和、操 作方便、简单高效等优点，并且避免了过渡金属催化剂 及光敏剂等昂贵试剂的使用.

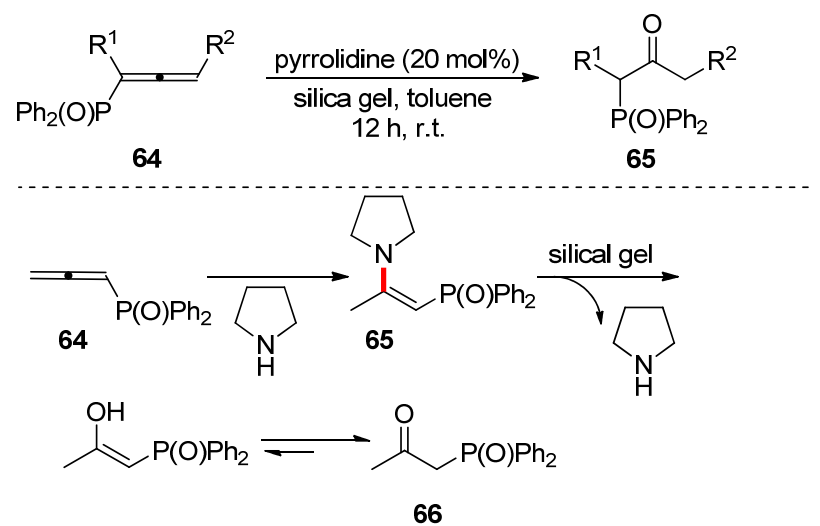

图式 14 二苯基膦联烯化合物合成 $\beta$-羰基膦酸酯化合物 Scheme 14 Synthesis of $\beta$-ketophosphine oxides from diphenylphosphorylallenes 
2019 年, Wang 等 ${ }^{[57]}$ 报道了在温和反应条件下以炔 基膦酸酯为原料, 苯甲醛肜化合物为羟基源试剂的合成 策略, 以中等到良好的收率制备了一系列 $\beta$-羊基膦氧化 物(Eq. 19). 该反应体系无需使用过渡金属催化剂, 在碱 性条件下炔基磷酸酯与苯甲醛肟即可发生反应，经过氧 杂迈克尔加成、消除一分子苯甲腈及最后的酸化异构化 后得到 $\beta$-羰基膦氧化物.

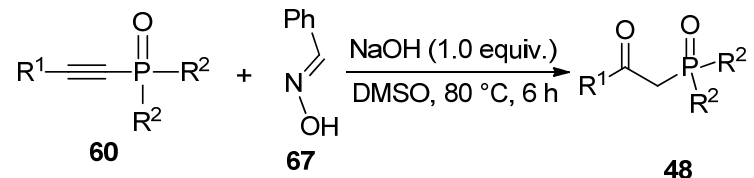

\section{3 总结与展望}

综上所述, 膦-氢化合物与不饱和烃的自由基加成 反应以及不饱和膦氧化合物的直接水合反应都为 $\beta$-羰 基膦氧化合物的制备提供了简单高效环境友好型的合 成途径. 但在该领域仍然有一部分工作需要科研工作者 去不断完善: 如在磷自由基引发过程中氧化剂非常重 要, 应该广泛开发以氧气为氧化剂的绿色氧化体系, 以 提升反应在工业上的应用前景. 在直接水合反应体系中 起始原料需要简单廉价易制备. 相信随着研究的不断深 入, $\beta$-羰基膦氧化合物合成路线必将更加经济绿色高效.

\section{References}

[1] (a) Perumal, S. K.; Adediran, S. A.; Pratt, R. F. Bioorg. Med. Chem. 2008, 16, 6987.

(b) Li, R.; Chen, X.; Wei, S.; Sun, K.; Fan, L.- L.; Liu, Y.; Qu, L.-B.; Zhao, Y.-F.; Yu, B. Adv. Synth. Catal. 2018, 360, 4807.

(c) Muhammad, M.-H.; Chen, X.-L.; Yu, B.; Qu, L.-B.; Zhao, Y.-F. Pure Appl. Chem. 2019, 91, 33.

(d) Jing, C.-F.; Chen, X.-L.; Sun, K.; Yang, Y.-K.; Chen, T.; Liu, Y.; Qu, L.-B.; Zhao, Y.-F.; Yu, B. Org. Lett. 2019, 21, 486.

(e) Liu, Y.-L.; Liang, Y.; Pi, S.-F.; Li, J.-H. J. Org. Chem. 2009, 74, 5691.

(f) Kondoh, A.; Yorimitsu, H.; Oshima, K. Chem.-Asian J. 2010, 5 , 398.

(g) Perumal, S.-K.; Adediran, S.-A.; Pratt, R.-F. Bioorg. Med. Chem. 2008, 16, 6987.

[2] (a) Yan, B.; Spilling, C.-D. J. Org. Chem. 2008, 73, 5385. (b) Pronin, S.-V.; Martinez, A.; Kuznedelov, K.; Severinov, K.; Shuman, H.-A.; Kozmin, S.-A. J. Am. Chem. Soc. 2011, 133, 12172 .

(c) Müller, S.; Mayer, T.; Sasse, F.; Maier, M.-E. Org. Lett. 2011, 13, 3940 .

[3] (a) Ryglowski, A.; Kafarski, P. Tetrahedron 1996, 52, 10685.

(b) Kitamura, M.; Tokunaga, M.; Noyori, R. J. Am. Chem. Soc. 1995, 117, 2931.

[4] McCabe, D.-J.; Duesler, E.-N.; Paine, R.-T. Inorg. Chem. 1985, 24, 4626.

[5] (a) Perumal, S.-K.; Adediran, S.-A.; Pratt, R.-F. Bioorg. Med. Chem. 2008, 16, 6987.

(b) Nguyen, L. M.; Diep, V. V.; Phan, H. T.; Niesor E. J.; Masson D.; GuyonGellin, Y.; Buattini, E.; Severi, C.; Azoulay, R.; Bentzen, C. L. WO 2004026242, 2004.

(c) Erion M. D.; Jiang, H.; Boyer, S. H. US 20060046980, 2006.

[6] (a) Arbuzov, B. A. Pure Appl. Chem. 1964, 9, 307. (b) Bhattacharya, A. K.; Thyagarajan, G. Chem. Rev. 1981, 81, 415.

[7] (a) Narkunan, K.; Nagarajan, M. J. Org. Chem. 1994, 59, 6386. (b) Maloney, K. M.; Chung, J. Y. L. J. Org. Chem. 2009, 74, 7574.

(c) Robert, R. M.; Ken, M.; Johann, C.; Jason, T.; Robert, L.; Margaret, F. Tetrahedron Lett. 2009, 50, 870.

(d) Demmer, C. S.; Krogsgaard-Larsen, N.; Bunch, L. Chem. Rev. 2011, $111,7981$.

[8] (a) Leca, D.; Fensterbank, L.; Lacote, E.; Malacria, M. Chem. Soc. Rev. 2005, 34, 858 .

(b) Marque, S.; Tordo, P. In New Aspects in Phosphorus Chemistry, 5th ed., Vol. 250, Ed.: Majoral, J. P., Springer, Berlin, Heidelberg, 2005, p. 43.

(c) Baumgartner, T.; Réau, R. Chem. Rev. 2006, 106, 4681. (d) Xie, J.-H.; Zhou, Q.-L. Acc. Chem. Res. 2008, 41, 581.

[9] Zhang, P.-B.; Zhang, L.-L.; Gao, Y.-Z.; Xu, J.; Fang, H.; Tang, G.; Zhao Y.- F. Chem. Commun. 2015, 51, 7839.

[10] Feng, S.-B.; Li, J.-L.; He, F.-F.; Li, T.; Li, H.-L.; Wang, X.-L.; Xie, X.-G.; She, X.-G. Org. Chem. Front. 2019, 6, 946.

[11] Gutierrez, V.; Mascaro, E.; Alonso, F.; Moglie, Y.; Radivoy, G. RSC Adv. 2015, 5, 65739 .

[12] Zhong, W.-W.; Zhang, Q.; Li, M.-S.; Hu, D.-Y.; Cheng, M.; Du, F.-T.; Ji, J.-X.; Wei, W. Synth. Commun. 2016, 46, 1377.

[13] Chen, X.; Chen, X.; Li, X.; Qu, C.; Qu, L.; Bi, W.; Sun, K.; Zhao, Y.-F. Tetrahedron 2017, 73, 2439

[14] Zeng, Y.-F.; Tan, D.-H.; Lv, W.-X.; Li, Q.-J.; Wang, H.-G. Eur. J. Org. Chem. 2015, 4335.

[15] Chen, X.; Li, X.; Chen, X.-L. Qu, L.-B.; Chen, J.-Y.; Sun, K.; Liu, Z.-D.; Bi, W.-Z.; Xia, Y.-Y.; Wu, H.-T.; Zhao, Y.-F. Chem. Commun. 2015, $51,3846$.

[16] Mi, X.; Wang, C.; Huang, M.; Zhang, J.; Wu, Y. Org. Lett. 2014, 16, 3356.

[17] Wang, L.-J.; Wang, A.-Q.; Xia, Y.; Wu, X.-X.; Liu, X.-Y.; Liang, Y.-M. Chem. Commun. 2014, 50, 13998.

[18] Zhou, Z.-Z.; Zheng, L.; Yan, X.-B.; Jin, D.-P.; He, Y.-T.; Liang, Y.-M. Org. Biomol. Chem. 2016, 14, 4507.

[19] Liu, J.; Zhao, S.; Song, W.; Li, R.; Guo, X.; Zhuo, K.; Yue, Y. Adv. Synth. Catal. 2017, 359, 609.

[20] Zhang, H.; Gu, Z.; Li, Z.; Pan, C.; Li, W.; Hu, H.; Zhu, C. J. Org. Chem. 2016, 81, 2122.

[21] Li, L.-L.; Huang, W.-B.; Chen, L.-J.; Dong, J.-X.; Ma, X.-B.; Peng, Y.-G. Angew. Chem., Int. Ed. 2017, 56, 10539.

[22] Yuan, J.-W.; Li, Y.-Z.; Mai, W.-P.; Yang, L.-R.; Qu, L.-B. Tetrahedron 2016, 72, 3084.

[23] Wei, W.; Ji, J.-X. Angew. Chem., Int. Ed. 2011, 50, 9097.

[24] Yang, X.-Y.; Du, F.-T.; Wei, W.; Ji, J.-X.; Chen, Q. Chin. J. Appl. Eviron. Biol. 2015, 21, 263.

[25] Yi, N.-N.; Wang, R.-J.; Zou, H.-X.; He, W.-B.; Fu, W.-Q.; He, W.-M. J. Org. Chem. 2015, 80, 5023.

[26] Zhou, M.-X.; Zhou, Y.; Song, Q.-L. Chem.-Eur. J. 2015, 21, 10654.

[27] Zhou, M.-X.; Chen, M.; Zhou, Y.; Yang, K.; Su, J.-H.; Du, J.-F.; Song, Q.-L. Org. Lett. 2015, 17, 1786.

[28] Zhou, Y.; Zhou, M.-X.; Chen, M.; Su, J.-H.; Du, J.-F.; Song, Q.-L. RSC Adv. 2015, 5, 103977.

[29] Zhou, Y.; Rao, C.-Q.; Mai, S.-Y.; Song, Q.-L. J. Org. Chem. 2016, 81, 2027.

[30] Gu, J.; Cai, C. Org. Biomol. Chem. 2017, 15, 4226.

[31] Ke, J.; Tang, Y.-L.; Yi, H.; Li, Y.-L.; Cheng, Y.-D.; Liu, C.; Lei, A.-W. Angew. Chem., Int. Ed. 2015, 54, 6604.

[32] Liang, W.; Zhang, Z.-J.; Yi, D.; Fu, Q.; Chen, S.-Y.; Yang, L.; Du, F.-T.; Ji, J.-X.; Wei, W. Chin. J. Chem. 2017, 35, 1378.

[33] Zhang, Z.-J.; Yi, D.; Fu, Q.; Liang, W.; Chen, S.-Y.; Yang, L.; Du, F.-T.; Ji, J.-X.; Wei, W. Tetrahedron Lett. 2017, 58, 2417.

[34] Fu, Q.; Yi, D.; Zhang, Z.-J.; Liang, W.; Chen, S.-Y. Yang, L.; Zhang, Q.; Ji, J.-X.; Wei, W. Org. Chem. Front. 2017, 4, 1385.

[35] Tang, L.; Wen, L.-X.; Sun, T.; Zhang, D.; Yang, Z.; Feng, C.-T.; Wang, Z.-Y. Asian J. Org. Chem. 2017, 6, 1683 .

[36] Mondal, M.; Bora, U. RSC Adv. 2013, 3, 18716.

[37] Pan, X. Q.; Zou, J. P.; Zhang, G. L.; Zhang, W. Chem. Commun. 
2010, 46, 1721.

[38] Zhou, J.; Zhang, G.-L.; Zou, J.-P.; Zhang, W. Eur. J. Org. Chem. 2011, 3412

[39] Zhou, P.; Jiang, Y.-J.; Zou, J.-P.; Zhang, W. Synthesis 2012, 44, 1043.

[40] Zhang, G.-Y.; Li, C.-K.; Li, D.-P.; Zeng, R.-S.; Shoberu, A.; Zou, J.-P. Tetrahedron 2016, 72, 2972.

[41] Zhou, P.; Hu, B.; Li, L.-D.; Rao, K.-R.; Yang, J.; Yu, F.-C. J. Org. Chem. 2017, 82, 13268.

[42] Tang, P.; Zhang, C.; Chen, E.; Chen, B.; Chen, W.; Yu, Y. Tetrahedron Lett. 2017, 58, 2157.

[43] Bu, M.-J.; Lu, G.-P.; Cai, C. Catal. Sci. Technol. 2016, 6, 413.

[44] Liu, D.; Chen, J.-Q.; Wang, X.-Z.; Xu, P.-F. Adv. Synth. Catal. 2017, 359, 2773.

[45] Qian, H.-F.; Li, C.-K.; Zhou, Z.-H.; Tao, Z.-K.; Shoberu, A.; Zou, J.-P. Org. Lett. 2018, 20, 5947.

[46] Shi, Y.; Chen, R.-S.; Guo, K.; Meng, F.; Cao, S.-J.; Gu, C.; Zhu, Y.-G. Tetrahedron Lett. 2018, 59, 2062.

[47] Peng, P.; Lu, Q.-Q.; Peng, L.; Liu, C.; Wang, G.-Y.; Lei, A.-W. Chem. Commun. 2016, 52, 12338.

[48] (a) Beller, M.; Seayad, J.; Tillack, A.; Jiao, H. Angew. Chem., Int. Ed. 2004, 43, 3368. (b) Zeng, X. Chem. Rev. 2013, 113, 6864.

[49] Sturtz, C. C. G.; Normant, H. Bull. Soc. Chim. Fr. 1966, 1707.

[50] Li, X.-B.; Hu, G.-B.; Luo, P.; Tang, G.; Gao, Y.-X.; Xu, P.-X.; Zhao, Y.-F. Adv. Synth. Catal. 2012, 354, 2427.

[51] Xie, L.-Y.; Yuan, R.; Wang, R.-J.; Peng, Z.-H.; Xiang, J.-N.; He, W.-M. Eur. J. Org. Chem. 2014, 13, 2668.

[52] Xiang, J.-N.; Yi, N.-N.; Wang, R.-J.; Lu, L.-H.; Zou, H.-X.; Pan, Y.; He, W.-M. Tetrahedron 2015, 71, 694.

[53] Liu, K.-J.; Liu, H.-M.; Wang, W.-G.; Ou, L.-J.; Wang, J.-J. Hu, B.-N. Chin. J. Org. Chem. 2014, 34, 2007 (in Chinese). (刘开建, 刘宏伟, 王文革, 欧丽娟, 王津津, 胡波年, 有机化学, 2014, 34, 2007.)

[54] Hou, J.-L.; Chen, Y.; Ma, D.-M.; Cordes, B.; Wang, J.-Y.; Wang, X.; Kühn, F.-E.; Guo, H.; Zhou, M.-D. Chem. Commun. 2015, 51, 7439.

[55] Zhu, Y.-G. Ph.D. Dissertation, Hunan University, Changsha, 2016 (in Chinese). (朱永刚, 博士论文, 湖南大学, 长沙, 2016.)

[56] Ruan, L.-H.; Wang, Q.; Sun, J.; Zhou, M.-D. Asian J. Org. Chem. 2018, 7, 1839 .

[57] Chen, L.-L.; Zhang, J.-W.; Yang, W.-W.; Chen, P.; Chen, D.-Y.; Wang, Y.-B. Org. Biomol. Chem. 2019, 17, 3003. 